\title{
Association between Dietary Energy Density and Mental Health in Overweight/Obese Women
}

\section{Niloofarsadat Maddahi}

Tehran University of Medical Sciences

\section{Habib Yarizadeh}

Tehran University of Medical Sciences

\section{Leila Setayesh}

TUMS

\section{Yasaman Nasir}

TUMS

\section{Shahab Alizadeh}

Tehran University of Medical Sciences

Khadijeh Mirzaei ( $\square$ mina_mirzaei101@yahoo.com )

\section{Research note}

Keywords: dietary energy density, mental health, stress, depression, anxiety, sleep quality

Posted Date: January 9th, 2020

DOI: https://doi.org/10.21203/rs.2.20529/v1

License: @ (i) This work is licensed under a Creative Commons Attribution 4.0 International License. Read Full License

Version of Record: A version of this preprint was published at BMC Research Notes on March 30th, 2020. See the published version at https://doi.org/10.1186/s13104-020-05025-1. 


\section{Abstract}

Objectives: Mental health, sleep quality and dietary intake are interlinked. Impairment of mental health and low sleep quality may contribute to obesity through the consumption of diets high in energy density. Nevertheless, it is not clear whether dietary energy density (DED) influences mental health. This study aimed to examine the association of DED with mental health indices, including depression, anxiety, stress, and sleep quality in overweight/obese women.

Results: After adjustment for age, BMI, and physical activity, subjects in the highest quartile of DED had higher systolic and diastolic blood pressure, but lower serum triglyceride, than those in the lowest quartile $(p<0.05)$. DED was significantly associated with increased odds of stress in the crude $(O R=2.15,95 \% \mathrm{Cl}$ : 1.01-4.56, $p=0.04)$ and adjusted model for age, $\mathrm{BMI}$, and physical activity $(\mathrm{OR}=2.56,95 \% \mathrm{Cl}: 1.13-5.79$, $\mathrm{p}=0.02$ ). No significant relationship was observed between DED and depression, anxiety and sleep quality.

\section{Introduction}

The prevalence of obesity has progressively risen in all parts of the world during the last decades and has become a main public health concern. It is well-known that the positive energy balance, usually resulted from excessive intake of energy, is the fundamental dietary factor associated with weight gain (1). Assessment of the overall impact of diet is commonly preferred for evaluation of single dietary constituents, such as energy intake. Among diet quality indices, dietary energy density (DED), as a measure of the whole diet, has been at the focus of many recent investigations (2). DED is a comparatively new dietary index that has an important role in body weight control (3), which is defined as the amount of energy per unit weight of a food or beverage, usually reported as kilocalories $/ 100 \mathrm{~g} \mathrm{(4)}$. High-energy dense diets are rich in fat, because dietary fat provides the greatest amount of energy per gram, but are low in vegetable, fruit and fiber $(2,5,6)$. It has been found that higher DED is associated with the risk of obesity (7) and obesity-related diseases (8), indicating that adopting diets with lower DED are important preventive approaches for obesity-related complications.

Furthermore, obesity is reported to be related to impaired mental health such as anxiety, stress, depression and low quality of sleep $(9,10)$; and on the other side, mental health, sleep behavior and dietary intake are interlinked (11). There is inadequate evidence in the emerging field of "relationship between dietary intake, mental health, and sleep quality". The majority of studies in this area of research have concentrated on food items, macronutrients, single nutrients or energy intakes $(12,13)$.

Nevertheless, limited research with conflicting results $(11,14-18)$ exists on the association between DED and indices of sleep quality and mental health. Thus, this study was performed to assed the relation of dietary energy density to mental health and sleep quality in women with overweight and obesity.

\section{Methods}




\section{Study population}

A total of 301 overweight/obese women took part in the present cross-sectional study. All subjects were randomly recruited from individuals referring to health centers in Tehran during 2016-2017. Inclusion criteria were age 18-56 years, being overweight or obese (body mass index (BMI) $\geq 25$ ), absence of any acute or chronic infection, no alcohol or drug abuse, no history of hypertension, and not being pregnant. Based on exclusion criteria, prospective subjects with a history of cardiovascular disease, cancer, sustained hypertension, diabetes, thyroid disease, cancer, acute or chronic infections, liver and kidney disease, and smokers were excluded from the study. Enrolment was voluntary, and the participants were informed about the objectives and all the stages of this study. Written informed consent was obtained from all participants before taking part in the study. The study protocol was approved by the local ethical committee of Tehran University of Medical Sciences (IR. TUMS.VCR.REC. 95-04-161-33893).

\section{Body composition and anthropometric measurement}

Body composition of all participants was mustered using a body composition analyzer (InBody770 scanner; InBody, Seoul, Korea) by following the manufacturer's protocol. Weight of the individuals was measured with the use of a digital scale (Seca, Hamburg, Germany) in light clothing and without shoes with precision near to $0.1 \mathrm{~kg}$. Height of participants was evaluated by a seca stadiometer, with exactness close to $0.1 \mathrm{~cm}$ in a standing position. BMI was calculated as weight $(\mathrm{kg}) / \mathrm{hieght}^{2}(\mathrm{~m})$. Furthermore, Waist (WC) and hip circumference (HC) were measured in the smallest girth and in the largest girth, respectively, with the use of an inelastic tape with accuracy nearest to $0.1 \mathrm{~cm}$.

\section{Evaluation of food intake and DED}

To assess dietary intake of participants, a semi-quantitative food frequency questionnaire (sq-FFQ) with 147 Iranian food items, containing a list of foods with standard serving sizes was used. The high reliability and validity and of the FFQ have been confirmed previously (19). All FFQ questionnaires were completed by trained nutritionists. The energy of food consumed was evaluated using Nutritionist 4 software (First Databank Inc., Hearst Corp., San Bruno, CA). DED was calculated by dividing the total dietary energy intake from consumed food $(\mathrm{kcal} / \mathrm{d})$ by the total weight $(\mathrm{g} / \mathrm{d})$ of consumed foods (excluding beverages) (20).

\section{Assessment of mental health and sleep quality}

Mental health was evaluated with the use of the 21-question version of the Depression Anxiety Stress Scales (DASS-21). The DASS-21 is a self-administered psychological questionnaire containing of 3 scales. Each subscale has 7 items based on 4-point Likert scale (0-3 scale) (Never, Sometimes, Often, Almost, Always)(21). The Pittsburgh Sleep Quality Index (PSQI) (22) was applied to subjectively measure the sleep quality of participants. Total scores could range from 0 to 21 , with global sum of " 5 " or greater indicates poor sleep quality (22). 
Blood samples were obtained from the forearm of all participants in the early morning between 8:00 and 10:00 am after a 10- to 12-hr overnight fasting. The samples were centrifuged at 3,500 rpm for $10 \mathrm{~min}$ at $-70 \mathrm{oC}$ and the separated sera stored at a temperature of $-80^{\circ} \mathrm{C}$. Serum concentrations of high-density lipoprotein cholesterol (HDL-C), total cholesterol (TC), triglyceride (TG), and low-density lipoprotein cholesterol (LDL-C) were evaluated by using of enzymatic approaches using related kits (Pars Azemun, Iran) and autoanalyzer system. Insulin level was assessed using an ELISA kit (Human insulin ELISA kit,DRG Pharmaceuticals, GmbH, Germany), and fasting concentration of glucose was measured using glucose oxidase method. Serum high-sensitive C-reactive protein (hs-CRP) was assessed with the use of immunoturbidimetric assay.

\section{Statistical analysis}

Analyses of continuous variables to assess differences among quartile of DED were performed using one-way analysis of variance (ANOVA). Analysis of Covariance (ANCOVA) was then used in order to find the difference between the means of investigated variables across quartiles of DED adjusted for age, physical activity and BMI. The logistic regression analysis was applied to find the relation of DED to sleep quality, stress, anxiety, and depression; this model was then adjusted for age, physical activity and BMI. The level of significance was set at a probability of $\leq 0.05$ for all tests. All statistical analyses were conducted using a statistical Package for Social Science (Version 22.0; SPSS Inc., Chicago IL, USA).

\section{Results}

A total of 301 women, aged 18-56 y, participated in this study and 293 subjects completed measurements. General characteristics of the study subjects are reported in Table 1. The mean age and BMI of participants were $36.39 \pm 8.41$ and $30.77 \pm 3.79$, respectively. There was a decreasing trend in serum TG across quartiles of DED (from Q1 to Q4) in the crude analysis and also after adjustment for age, BMI, and physical activity ( $\mathrm{p} \leq 0.05)$. Moreover, after adjustment for age, BMI, and physical activity, subjects in the highest quartile of DED had higher systolic $(p=0.01)$ and diastolic blood pressure $(p=0.04)$ than those in the lowest quartile. No significant differences were observed in age, total cholesterol, HDL, LDL, hs-CRP, insulin, HOMA-IR, FBS, depression, anxiety, stress, sleep quality, body composition, and anthropometric indices across the categories of DED (Table 2).

DED was associated with a 2.15-fold increased odds of stress (OR=2.15, 95\%Cl:1.01-4.56, $p=0.04)$, and even after adjusting for age, $\mathrm{BMI}$, and physical activity this association remained statistically significant $(\mathrm{OR}=2.56,95 \% \mathrm{Cl}: 1.13-5.79, \mathrm{p}=0.02)$. No significant association was found between DED and depression, anxiety and sleep quality (Table 3 ).

\section{Discussion}


This study aimed to investigate the association between dietary energy density, a marker of dietary quality, with mental health of overweight/obese women. After adjustment for potential covariates, higher DED was significantly related to 2.56 -fold increased odds of stress. Although, no significant relationship was found between DED with anxiety, depression and sleep quality.

In line with our finding, Heath et al. (23) found that higher levels of stress are associated with a higher energy intake. Under stressful conditions, humans desire palatable foods that are energy dense (24), specially elevated eating of high-sugar, high-fat foods and processed foods (25-28) and a decrease in consumption of main meals, fruits and vegetables $(26,28,29)$. Moreover, chronic stress was reported to be related to empty calories (added solid fat and sugar) consumption and evening intake of added sugars (30). The preference of highly palatable, energy-dense food items is attributed to hormones secreted in stress response, such as cortisol (31). Behaviorally, it has been suggested that during stress people have less energy and time to devote to the preparation of foods; thus, they have an elevated dependence on pre-processed convenience food items, which are frequently rich in energy $(32,33)$. The 'comfort food hypothesis' proposes that chronic stress could endorse a coping strategy resulting in higher intake of macronutrient and preference towards food comprising more carbohydrates and saturated fats (29), which have higher energy density.

The western, high fat-high sugar, and sweet dietary patterns have been reported to be related to higher odds for depression (34-37); though, some studies did not reveal such a relationship (38). In the study by Grossniklaus et al. (39), increased depressive symptoms, independently predicted increased food and beverage energy density, and explained variance above that explained by male gender, younger age, and reporting adequate caloric intake. There is only one study exploring the relation of DED to depression, which consistent with our study, found no association between DED and depression (40). For anxiety, prior studies found that the saturated fat and added sugars dietary pattern is significantly related to higher anxiety, but in agreement with our results, no association between anxiety level and energy intake was detected (41). Other recent investigations showed that diets high in sugars and fats are associated with higher anxiety level via changes of protein, glucose, and energy homeostasis, and increases in corticosterone and inflammatory cytokines $(42,43)$.

Some studies, in agreement with the current study, found no association between DED and sleep duration (15), but the majority of studies shave identified that because of the elevated feeling of appetite and hunger, people with low sleep quality or short sleep duration have a higher DED or total daily energy intake and preferred food items with high content of carbohydrates and fats, compared with individuals with adequate sleep duration $(11,16)$. Mechanistically, sleep modulates the pattern of secretion of two key hormones involved in appetite and energy regulation: ghrelin and leptin (44). Specifically, partial sleep deprivation appears to lead to increased serum ghrelin and reduced serum leptin, both of which result in elevated appetite (45). One likely justification for the lack of link between sleep quality and DED in the present study may be that compared with other study populations, our participants differed in some characteristics. For example, our samples were restricted to women and BMI, as an important factor affecting sleep quality, differed significantly between previous studies and our study. 


\section{Conclusion}

In conclusion, this study provides the first evidence that DED is significantly associated with stress. Additional large well-designed studies should be conducted to elucidate the relation of dietary energy density to mental health to reach to a robust conclusion.

\section{Limitation}

Some limitations of the current study should be considered. Briefly, this observational investigation might have potential recall bias because of its cross-sectional design, and consequently, causal inferences could not be extracted. There may be some factors, such as socioeconomic or education level of subjects, affecting the DED and mental health that were not measured in the present study. Demographic and mental health data were self-reported, and these data might be subject to social desirability bias and under or over reporting. Finally, the study population were restricted to women, which this limits generalizability of findings to men; therefore, replication of our results with use of larger samples in both sexes is essential.

\section{Declarations}

Ethics approval (Grant ID: IR.TUMS.VCR.REC 95-04-161-33893)

Availability of data and materials

No funding

\section{Conflict of interest}

None

All authors declared that they have no competing interests

Acknowledgments: We would like to thank the Tehran University of Medical Sciences. This work was supported financially by the Tehran University of Medical Sciences (TUMS), Tehran, Iran (Grant ID: 95-04161-33893).

Author Contribution: "YN, and LS designed research; SHA conducted research; HY analyzed data; NSM wrote the paper; KhM had primary responsibility for final content. All authors read and approved the final manuscript."

Consent for publication: This is formally to submit the article entitled "Association between Dietary Energy Density and Mental Health in Overweight/Obese Women" prepared by the Tehran University of Medical Sciences for review and, hopefully, publication in your prestigious journal. The authors would like to advise that all authors listed have contributed to the work. All authors have agreed to submit the 
manuscript to BMC Research Notes. No part of the work has been published before. There is no conflict of interest in this paper.

\section{Abbreviations}

(DED) dietary energy density

(BMI) body mass index

(WC) waist circumference

(HC) hip circumference

(FFQ) food frequency questionnaire

21-question version of the Depression Anxiety Stress Scales (DASS-21).

The Pittsburgh Sleep Quality Index (PSQI)

high-density lipoprotein cholesterol (HDL-C)

cholesterol (TC)

triglyceride (TG)

low-density lipoprotein cholesterol (LDL-C)

Serum high-sensitive C-reactive protein (hs-CRP)

one-way analysis of variance (ANOVA)

Analysis of Covariance (ANCOVA)

\section{References}

1. Apolzan JW, Bray GA, Hamilton MT, Zderic TW, Han H, Champagne CM, et al. Short-term overeating results in incomplete energy intake compensation regardless of energy density or macronutrient composition. Obesity. 2014;22(1):119-30.

2. Rouhani MH, Haghighatdoost F, Surkan PJ, Azadbakht L. Associations between dietary energy density and obesity: A systematic review and meta-analysis of observational studies. Nutrition. 2016;32(10):1037-47.

3. Pérez-Escamilla R, Obbagy JE, Altman JM, Essery EV, McGrane MM, Wong YP, et al. Dietary energy density and body weight in adults and children: a systematic review. Journal of the Academy of Nutrition and Dietetics. 2012;112(5):671-84. 
4. Aburto TC, Cantoral A, Hernández-Barrera L, Carriquiry AL, Rivera JA. Usual dietary energy density distribution is positively associated with excess body weight in Mexican children. The Journal of nutrition. 2015;145(7):1524-30.

5. Piernas C, Popkin BM. Increased portion sizes from energy-dense foods affect total energy intake at eating occasions in US children and adolescents: patterns and trends by age group and sociodemographic characteristics, 1977-2006. The American journal of clinical nutrition. 2011;94(5):1324-32.

6. McNaughton SA, Wattanapenpaiboon N, Wark JD, Nowson CA. An energy-dense, nutrient-poor dietary pattern is inversely associated with bone health in women. The Journal of nutrition. 2011;141(8):1516-23.

7. Cedillo YE, Garr-Barry V, Maciel B, Fernández JR. Dietary Energy-Density and Adiposity Markers Among a Cohort of Multi-ethnic Children. Maternal and child health journal. 2019:1-11.

8. Vernarelli J, DiSarro R. Eat Better, Run Longer? Dietary Energy Density Is Associated with Cardiovascular Fitness Level in a Nationally Representative Sample of Adolescents (P16-030-19). Oxford University Press; 2019.

9. Marshall NS, Glozier N, Grunstein RR. Is sleep duration related to obesity? A critical review of the epidemiological evidence. Sleep medicine reviews. 2008;12(4):289-98.

10. Dimov S, Mundy LK, Bayer JK, Jacka FN, Canterford L, Patton GC. Diet quality and mental health problems in late childhood. Nutritional neuroscience. 2019:1-9.

11. Stelmach-Mardas M, Iqbal K, Mardas M, Schwingshackl L, Walkowiak J, Tower R, et al. Synchronic inverse seasonal rhythmus of energy density of food intake and sleep quality: a contribution to chrono-nutrition from a Polish adult population. European journal of clinical nutrition. 2017;71(6):718.

12. Dashti HS, Scheer FA, Jacques PF, Lamon-Fava S, Ordovás JM. Short sleep duration and dietary intake: epidemiologic evidence, mechanisms, and health implications. Advances in nutrition. 2015;6(6):648-59.

13. Weiss A, Xu F, Storfer-Isser A, Thomas A, levers-Landis CE, Redline S. The association of sleep duration with adolescents' fat and carbohydrate consumption. Sleep. 2010;33(9):1201-9.

14. Haghighatdoost F, Karimi G, Esmaillzadeh A, Azadbakht L. Sleep deprivation is associated with lower diet quality indices and higher rate of general and central obesity among young female students in Iran. Nutrition (Burbank, Los Angeles County, Calif). 2012;28(11-12):1146-50.

15. Onvani S, Najafabadi MM, Haghighatdoost F, Larijani B, Azadbakht L. Short sleep duration is related to kidney-related biomarkers, but not lipid profile and diet quality in diabetic nephropathy patients. International Journal for Vitamin and Nutrition Research. 2019.

16. Kjeldsen JS, Hjorth MF, Andersen R, Michaelsen KF, Tetens I, Astrup A, et al. Short sleep duration and large variability in sleep duration are independently associated with dietary risk factors for obesity in Danish school children. International journal of obesity. 2014;38(1):32. 
17. Hjorth M, Quist J, Andersen R, Michaelsen K, Tetens I, Astrup A, et al. Change in sleep duration and proposed dietary risk factors for obesity in D anish school children. Pediatric obesity. 2014;9(6):e156e9.

18. Grossniklaus DA, Dunbar SB, Gary R, Tohill BC, Frediani JK, Higgins MK. Dietary energy density: a mediator of depressive symptoms and abdominal obesity or independent predictor of abdominal obesity? European Journal of Cardiovascular Nursing. 2012;11(4):423-31.

19. Esmaillzadeh A, Mirmiran P, Azizi F. Whole-grain intake and the prevalence of hypertriglyceridemic waist phenotype in Tehranian adults. The American journal of clinical nutrition. 2005;81(1):55-63.

20. Kant AK, Graubard BI. Energy density of diets reported by American adults: association with food group intake, nutrient intake, and body weight. International Journal of Obesity. 2005;29(8):950.

21. Henry JD, Crawford JR. The short-form version of the Depression Anxiety Stress Scales (DASS-21): Construct validity and normative data in a large non-clinical sample. British journal of clinical psychology. 2005;44(2):227-39.

22. Buysse DJ, Reynolds III CF, Monk TH, Berman SR, Kupfer DJ. The Pittsburgh Sleep Quality Index: a new instrument for psychiatric practice and research. Psychiatry research. 1989;28(2):193-213.

23. Heath G, Dorrian J, Coates A. Associations between shift type, sleep, mood, and diet in a group of shift working nurses. Scandinavian journal of work, environment \& health. 2019.

24. Torres SJ, Nowson CA. Relationship between stress, eating behavior, and obesity. Nutrition (Burbank, Los Angeles County, Calif). 2007;23(11-12):887-94.

25. Oliver G, Wardle J, Gibson EL. Stress and food choice: a laboratory study. Psychosomatic medicine. 2000;62(6):853-65.

26. Mikolajczyk RT, El Ansari W, Maxwell AE. Food consumption frequency and perceived stress and depressive symptoms among students in three European countries. Nutrition Journal. 2009;8(1):31.

27. Ng DM, Jeffery RW. Relationships between perceived stress and health behaviors in a sample of working adults. Health psychology. 2003;22(6):638.

28. O'Connor DB, Jones F, Conner M, McMillan B, Ferguson E. Effects of daily hassles and eating style on eating behavior. Health Psychology. 2008;27(1S):S20.

29. Singh A, Trumpff C, Genkinger J, Davis A, Spann M, Werner E, et al. Micronutrient dietary intake in Latina pregnant adolescents and its association with level of depression, stress, and social support. Nutrients. 2017;9(11):1212.

30. Widaman AM, Witbracht MG, Forester SM, Laugero KD, Keim NL. Chronic stress is associated with indicators of diet quality in habitual breakfast skippers. Journal of the Academy of Nutrition and Dietetics. 2016;116(11):1776-84.

31. Takeda E, Terao J, Nakaya Y, Miyamoto K-i, Baba Y, Chuman H, et al. Stress control and human nutrition. The Journal of Medical Investigation. 2004;51(3, 4):139-45.

32. Prentice AM, Jebb SA. Fast foods, energy density and obesity: a possible mechanistic link. Obesity reviews. 2003;4(4):187-94. 
33. Tseng M, Fang CY. Stress is associated with unfavorable patterns of dietary intake among female chinese immigrants. Annals of Behavioral Medicine. 2011;41(3):324-32.

34. Akbaraly TN, Brunner EJ, Ferrie JE, Marmot MG, Kivimaki M, Singh-Manoux A. Dietary pattern and depressive symptoms in middle age. The British Journal of Psychiatry. 2009;195(5):408-13.

35. Vermeulen E, Stronks K, Snijder MB, Schene AH, Lok A, De Vries JH, et al. A combined high-sugar and high-saturated-fat dietary pattern is associated with more depressive symptoms in a multi-ethnic population: The helius (healthy life in an urban setting) study. Public health nutrition. 2017;20(13):2374-82.

36. Sánchez-Villegas A, Verberne L, De Irala J, Ruiz-Canela M, Toledo E, Serra-Majem L, et al. Dietary fat intake and the risk of depression: the SUN Project. PloS one. 2011;6(1):e16268.

37. Sánchez-Villegas A, Toledo E, De Irala J, Ruiz-Canela M, Pla-Vidal J, Martínez-González MA. Fastfood and commercial baked goods consumption and the risk of depression. Public health nutrition. 2012;15(3):424-32.

38. Chocano-Bedoya PO, O'Reilly EJ, Lucas M, Mirzaei F, Okereke OI, Fung TT, et al. Prospective study on long-term dietary patterns and incident depression in middle-aged and older women. The American journal of clinical nutrition. 2013;98(3):813-20.

39. Grossniklaus DA, Dunbar SB, Tohill BC, Gary R, Higgins MK, Frediani J. Psychological factors are important correlates of dietary pattern in overweight adults. The Journal of cardiovascular nursing. 2010;25(6):450.

40. Atlantis E, Lange K, Goldney RD, Martin S, Haren MT, Taylor A, et al. Specific medical conditions associated with clinically significant depressive symptoms in men. Social psychiatry and psychiatric epidemiology. 2011;46(12):1303-12.

41. Masana MF, Tyrovolas S, Kolia N, Chrysohoou C, Skoumas J, Haro JM, et al. Dietary Patterns and Their Association with Anxiety Symptoms among Older Adults: The ATTICA Study. Nutrients. 2019;11(6):1250.

42. Bakhtiyari M, Ehrampoush E, Enayati N, Joodi G, Sadr S, Delpisheh A, et al. Anxiety as a consequence of modern dietary pattern in adults in Tehran-Iran. Eating behaviors. 2013;14(2):107-12.

43. Dutheil S, Ota KT, Wohleb ES, Rasmussen K, Duman RS. High-fat diet induced anxiety and anhedonia: impact on brain homeostasis and inflammation. Neuropsychopharmacology. 2016;41(7):1874.

44. Morselli LL, Guyon A, Spiegel K. Sleep and metabolic function. Pflügers Archiv-European Journal of Physiology. 2012;463(1):139-60.

45. Shlisky JD, Hartman TJ, Kris-Etherton PM, Rogers CJ, Sharkey NA, Nickols-Richardson SM. Partial sleep deprivation and energy balance in adults: an emerging issue for consideration by dietetics practitioners. Journal of the Academy of Nutrition and Dietetics. 2012;112(11):1785-97.

\section{Tables}

Table 1. Characteristics of the study participants 


\begin{tabular}{|c|c|c|c|}
\hline Variable & Mean \pm SD & Minimum & Maximum \\
\hline Age (years) & $36.39 \pm 8.41$ & 18.00 & 56.00 \\
\hline Body weight $(\mathrm{Kg})$ & $80.22 \pm 11.28$ & 59.50 & 122.40 \\
\hline BMI $\left(\mathrm{Kg} / \mathrm{m}^{2}\right)$ & $30.77 \pm 3.79$ & 25.00 & 40.70 \\
\hline Total Cholesterol $(\mathrm{mg} / \mathrm{dl})$ & $183.78 \pm 35.33$ & 104.00 & 344.00 \\
\hline TG $(\mathrm{mg} / \mathrm{dl})$ & $120.78 \pm 68.69$ & 37.00 & 512.00 \\
\hline HDL $(\mathrm{mg} / \mathrm{dl})$ & $46.36 \pm 10.64$ & 18.00 & 82.00 \\
\hline LDL $(\mathrm{mg} / \mathrm{dl})$ & $94.41 \pm 24.05$ & 34.00 & 156.00 \\
\hline SBP $(\mathrm{mmHg})$ & $111.24 \pm 13.41$ & 76.00 & 159.00 \\
\hline DBP $(\mathrm{mmHg})$ & $77.69 \pm 9.61$ & 51.00 & 111.00 \\
\hline hs-CRP $(\mathrm{mg} / \mathrm{L})$ & $4.25 \pm 4.59$ & 00.00 & 22.73 \\
\hline Insulin $(\mu \mathrm{IU} / \mathrm{mL})$ & $15.59 \pm 6.02$ & 6.67 & 65.89 \\
\hline HOMA & $3.40 \pm 1.52$ & 1.29 & 16.59 \\
\hline FBS $(\mathrm{mmol} / \mathrm{L})$ & $4.85 \pm 0.53$ & 3.72 & 7.61 \\
\hline BFM $(\mathrm{Kg})$ & $33.52 \pm 7.74$ & 19.40 & 59.40 \\
\hline FFM $(\mathrm{Kg})$ & $46.68 \pm 5.50$ & 35.30 & 67.70 \\
\hline WC $(\mathrm{cm})$ & $98.56 \pm 9.43$ & 80.10 & 131.30 \\
\hline Depression & $5.23 \pm 4.69$ & 00.00 & 20.00 \\
\hline anxiety & $5.08 \pm 3.98$ & 00.00 & 19.00 \\
\hline stress & $7.87 \pm 5.02$ & 00.00 & 21.00 \\
\hline PSQI & $5.65 \pm 3.44$ & 00.00 & 21.00 \\
\hline DED & $1.23 \pm 0.34$ & 0.61 & 3.79 \\
\hline & & & \\
\hline & & & \\
\hline & & & \\
\hline & & & \\
\hline & & & \\
\hline & & & \\
\hline & & & \\
\hline & & & \\
\hline & & & \\
\hline & & & \\
\hline
\end{tabular}

SD: Standard deviation; BMI: Body mass index; TG: Triglyceride; LDL: Low density lipoprotein; HDL; High density lipoprotein; FBS: fasting blood sugar; SBP: Systolic blood pressure; DBP: Diastolic Blood Pressure; BFM: body fat mas; FFM: fat free mass; hs-CRP, high-sensitivity $C$-reactive protein; HOMA-IR, homeostatic model assessment of insulin resistance

Table 2. Mean and SD of anthropometric body composition, Blood parameters and Blood pressure across the quartiles of DED. 


\begin{tabular}{|c|l|l|l|l|l|l|}
\hline Variable & \multicolumn{1}{|c|}{ Q1 } & \multicolumn{1}{|c|}{ Q2 } & \multicolumn{1}{|c|}{ Q3 } & \multicolumn{1}{|c|}{ Q4 } & \\
\hline Age (years) & $38.20 \pm 7.58$ & $35.90 \pm 8.66$ & $35.73 \pm 9.27$ & $36.16 \pm 8.41$ & 0.28 & $0.25 \mathrm{a}$ \\
\hline Body weight (Kg) & $79.46 \pm 11.24$ & $80.62 \pm 11.28$ & $81.07 \pm 10.71$ & $78.88 \pm 10.89$ & 0.62 & $0.74 \mathrm{~b}$ \\
\hline BMI $\left(\mathrm{Kg} / \mathrm{m}^{2}\right)$ & $30.48 \pm 3.46$ & $30.95 \pm 3.96$ & $30.88 \pm 3.67$ & $30.76 \pm 3.94$ & 0.89 & $0.92 \mathrm{~b}$ \\
\hline $\begin{array}{c}\text { Total Cholesterol } \\
(\mathrm{mg} / \mathrm{dl})\end{array}$ & $183.85 \pm 32.57$ & $184.91 \pm 37.12$ & $182.92 \pm 34.89$ & $180.98 \pm 38.93$ & 0.94 & $0.87 \mathrm{c}$ \\
\hline TG (mg/dl) & $117.04 \pm 58.96$ & $115.82 \pm 67.28$ & $147.03 \pm 94.78$ & $103.17 \pm 44.16$ & 0.006 & $0.001 \mathrm{c}$ \\
\hline HDL $(\mathrm{mg} / \mathrm{dl})$ & $46.04 \pm 10.28$ & $47.03 \pm 12.56$ & $45.89 \pm 10.15$ & $47.44 \pm 9.50$ & 0.83 & $0.50 \mathrm{c}$ \\
\hline LDL $(\mathrm{mg} / \mathrm{dl})$ & $93.70 \pm 24.23$ & $93.91 \pm 24.54$ & $97.33 \pm 23.09$ & $91.08 \pm 24.56$ & 0.58 & $0.48 \mathrm{c}$ \\
\hline SBP (mmHg) & $109.35 \pm 14.35$ & $111.14 \pm 13.67$ & $109.73 \pm 12.49$ & $114.72 \pm 13.25$ & 0.09 & $0.01 \mathrm{c}$ \\
\hline DBP (mmHg) & $76.17 \pm 9.25$ & $76.89 \pm 9.07$ & $77.31 \pm 9.75$ & $80.12 \pm 10.30$ & 0.09 & $0.04 \mathrm{c}$ \\
\hline hs-CRP (mg/L) & $4.12 \pm 4.92$ & $4.39 \pm 4.50$ & $3.63 \pm 4.06$ & $4.74 \pm 5.01$ & 0.62 & $0.55 \mathrm{c}$ \\
\hline Insulin ( $\mu \mathrm{IU} / \mathrm{mL})$ & $14.69 \pm 4.97$ & $15.02 \pm 4.97$ & $16.76 \pm 6.28$ & $15.62 \pm 7.77$ & 0.26 & $0.44 \mathrm{c}$ \\
\hline HOMA-IR & $3.17 \pm 1.25$ & $3.35 \pm 1.30$ & $3.57 \pm 1.48$ & $3.45 \pm 2.03$ & 0.56 & $0.71 \mathrm{c}$ \\
\hline FBS (mmol/L) & $4.83 \pm 0.52$ & $4.88 \pm 0.52$ & $4.80 \pm 0.52$ & $4.89 \pm 0.58$ & 0.79 & $0.63 \mathrm{c}$ \\
\hline BFM (Kg) & $32.65 \pm 7.47$ & $33.88 \pm 7.79$ & $33.81 \pm 7.51$ & $33.48 \pm 8.12$ & 0.77 & $0.94 \mathrm{~b}$ \\
\hline FFM (Kg) & $46.62 \pm 5.58$ & $47.04 \pm 5.40$ & $47.02 \pm 5.70$ & $45.83 \pm 5.10$ & 0.52 & $0.61 \mathrm{~b}$ \\
\hline WC (cm) & $97.60 \pm 9.72$ & $99.14 \pm 9.74$ & $99.10 \pm 8.91$ & $98.05 \pm 9.41$ & 0.71 & $0.82 \mathrm{~b}$ \\
\hline Depression & $4.84 \pm 4.77$ & $5.35 \pm 4.83$ & $5.57 \pm 4.80$ & $4.94 \pm 4.30$ & 0.78 & $0.73 \mathrm{c}$ \\
\hline anxiety & $5.40 \pm 4.17$ & $5.25 \pm 3.75$ & $5.07 \pm 3.87$ & $4.31 \pm 3.96$ & 0.38 & $0.55 \mathrm{c}$ \\
\hline stress & $7.40 \pm 4.76$ & $8.22 \pm 5.24$ & $7.92 \pm 5.11$ & $7.86 \pm 5.06$ & 0.82 & $0.72 \mathrm{c}$ \\
\hline PSQI & $6.09 \pm 3.87$ & $5.41 \pm 3.21$ & $5.62 \pm 3.32$ & $5.31 \pm 3.08$ & 0.66 & $0.65 \mathrm{c}$ \\
\hline
\end{tabular}

SD: Standard deviation; BMI: Body mass index; TG: Triglyceride; LDL: Low density lipoprotein; HDL; High density lipoprotein; FBS: fasting blood sugar; SBP: Systolic blood pressure; DBP: Diastolic Blood Pressure; BFM: body fat mas; FFM: fat free mass; hs-CRP, high-sensitivity $C$ reactive protein; HOMA-IR, homeostatic model assessment of insulin resistance

$\beta$ : Adjusted model. a, adjusted for BMI and physical activity; $b$, adjusted for age and physical activity; $c$; adjusted for age BMI and physical activity;

Table 3. Logistic regression analysis for the association of DED with PSQI, depression, anxiety, and stress.

\begin{tabular}{|l|l|l|l|}
\hline & & OR & P \\
\hline PSQI & Model 1 & $0.50(0.20$ to 1.25$)$ & 0.14 \\
\cline { 2 - 4 } & Model 2 & $0.52(0.20$ to 1.33$)$ & 0.17 \\
\hline Depression & Model 1 & $0.88(0.40$ to 1.94$)$ & 0.76 \\
\cline { 2 - 4 } & Model 2 & $0.98(0.44$ to 2.18$)$ & 0.96 \\
\hline \multirow{3}{*}{ Anxiety } & Model 1 & $0.53(0.21$ to 1.32$)$ & 0.17 \\
\cline { 2 - 4 } & Model 2 & $0.59(0.22$ to 1.52$)$ & 0.27 \\
\hline Stress & Model 1 & $2.15(1.01$ to 4.56$)$ & 0.04 \\
\cline { 2 - 4 } & Model 2 & $2.56(1.13$ to 5.79$)$ & 0.02 \\
\hline
\end{tabular}


Model 1, crude mode l; model 2, adjusted for age, BMI and physical activity. 